\title{
Volume and Surface Field Coupling on Complex Electrodynamic Surfaces for MM-Wave and THz Applications
}

\author{
A. J. MacLachlan, C. W. Robertson, A. W. Cross, K. Ronald, and A. D. R. Phelps \\ Department of Physics, SUPA, University of Strathclyde, \\ Glasgow, G4 0NG, Scotland, UK \\ a.d.r.phelps@strath.ac.uk
}

\begin{abstract}
A theoretical analysis is presented of the coupling of volume and surface fields in electromagnetic structures, facilitated by two dimensional complex electrodynamic surfaces. This theory is applicable over a broad frequency range from $\mathrm{mm}$-wave frequencies through $\mathrm{THz}$ to even optical frequencies. This analysis is fundamental to some of the routes to the innovation of high power, mm-wave and $\mathrm{THz}$ sources, solar cells and novel subwavelength absorbers.
\end{abstract}

Keywords-coupling, two dimensional periodic lattices, metadielectric, mm-wave, $\mathrm{THz}$ applications

\section{INTRODUCTION}

One and two dimensional periodic structures have been applied successfully as Bragg reflectors in a mm-wave free electron laser [1], i.e. in a 'fast wave' source. The present work has been stimulated by the potential of two dimensional periodic structures to facilitate the realization of highly overmoded high power 'slow wave' (Cherenkov) sources of electromagnetic radiation. The results are also applicable to a range of other electromagnetic applications including to solar cells and novel subwavelength absorbers. A feature of electromagnetic cavities bounded by two dimensional periodic lattice structures (PSLs) is their capacity to support both volume and surface fields. Under optimum conditions, the PSLs facilitate the coupling of volume and surface fields to produce a single high-Q eigenmode [2-6]. When the dimension transverse to the two dimensional surface structure is less that an electromagnetic wavelength the PSL can acquire the characteristics of a 'metadielectric' [2]. Interaction of this 'effective metadielectric' eigenmode, for example with an appropriate electron beam, is fundamental to the innovation of high power radiation sources, with particular relevance to the $\mathrm{mm}$-wave and $\mathrm{THz}$ regions of the spectrum. The frequency range for which the theory is valid extends to an even broader range of frequencies than the mmwave and THz regions and the applications in addition to high power radiation sources also include novel sub-wavelength absorbers and solar cells.

\section{THEORY}

The PSL's eigenfield for the case of an oversized cylindrical PSL can be described as a superposition of a well-defined $T M_{0, T}$ volume field and high order $T M_{\bar{m}, n}$ surface fields. This theory is also applicable to planar PSLs based on the assumption that the mean radius of the cylinder $r_{0}$ is large in

AFOSR provided support under award numbers FA8655-13-1-2132 and FA9550-17-1-0095. comparison to the operating wavelength $\left(r_{0} \gg \lambda\right)$, which has been made throughout this analysis. Lattice synchronization is obtained by launching a near cut-off, symmetric $T M_{0, T}$ volume mode (where $\mathrm{T}$ and 0 are the number of radial and azimuthal variations respectively) through the structure, or in the case of planar geometry, by exciting a Fabry-Perot standing plane wave that exists between the reflecting surfaces due to scattering of the surface currents. The cylindrical PSL can be substituted for a smooth waveguide with a fictitious magnetic surface current density $\boldsymbol{j}_{m}$ (bold font symbols indicate 3 -vectors) in place of the corrugation to describe field coupling or scattering. Assuming $r_{0}$ is large, the theory developed for a cylindrical PSL is also relevant to the study of an equivalent planar PSL. From Maxwell's curl equations,

$$
\nabla^{2} \boldsymbol{H}=-\omega^{2} \varepsilon \mu \boldsymbol{H}+i \omega \varepsilon \boldsymbol{j}_{m}
$$

where $\varepsilon=\varepsilon_{r} \varepsilon_{0}$ and $\mu=\mu_{r} \mu_{0}$ denote respectively the permittivity and permeability in the 'effective metadielectric' material. In the theoretical study we take $\varepsilon_{r}=1$ and $\mu_{r}=1$ in the case of vacuum. When $r_{0} \gg \lambda_{o p}$ and providing the corrugation $\Delta r$ is suitably shallow $\left(\Delta r \ll \lambda_{o p}\right)$ the PSL's eigenfield can be described as a superposition of waveguide modes (where the volume and surface modes have real and imaginary transverse wavenumbers respectively). The transverse magnetic $\boldsymbol{H}$ and electric $\boldsymbol{E}$ fields are expanded as a sum of the waveguide modes $\boldsymbol{H}, \boldsymbol{E}=\sum_{q}\left(C_{q}(z), D_{q}(z)\right)\left(\boldsymbol{H}_{q}, \boldsymbol{E}_{q}\right)$, allowing the field inside the finite cylinder to be described as a slow wave envelope filled with fast varying terms. The field inside the structure is decomposed as a set of eigenfields, where $C_{q}(z)$ and $D_{q}(z)$ are the slowly varying magnetic and electric eigenfield amplitudes respectively

$$
\begin{aligned}
& \sum_{q} \nabla^{2}\left(C_{q}(z) \boldsymbol{H}_{\boldsymbol{q}}\right)=-\omega^{2} \varepsilon \mu \sum_{q} C_{q}(z) \boldsymbol{H}_{q}+i \omega \varepsilon \boldsymbol{j}_{m} \\
& \sum_{q} \nabla^{2}\left(D_{q}(z) \boldsymbol{E}_{q}\right)=-\omega^{2} \varepsilon \mu \sum_{q} D_{q}(z) \boldsymbol{E}_{q}
\end{aligned}
$$

Eqs. (1a) and (1b) describe the structure's fields in their general form and volume and surface field amplitudes are introduced later. To describe succinctly the field distribution inside it is sufficient to define only the magnetic fields as the electric fields can be derived from them. The total power transmitted through the structure is defined by integrating the Poynting vector $\frac{1}{2}\left(\boldsymbol{E} \times \boldsymbol{H}^{*}\right)$ over the cross section. This treatment is restricted to consider only the near cut-off volume mode $\left(\omega \approx \omega_{0}^{v}\right)$ for which $k_{z} \rightarrow 0$ and $\lambda_{z} \rightarrow \infty$. In the case of a planar system, the structure is assumed to be 
infinitely large and edge effects are neglected. In practice, however, some detuning from the ideal situation where $\omega=$ $\omega_{0}$ exists due to the structure's finite length. $\boldsymbol{E} \times \boldsymbol{H}^{*}$ is implicitly defined by multiplying both sides of Eq.(1a) by the complex conjugate of the transverse magnetic cut-off mode $\left(\boldsymbol{H}_{q^{\prime}}^{*}\right)$. Separating $\nabla^{2}$ into its transverse and longitudinal components and substituting the Helmholtz equation $\nabla_{\perp}^{2} \boldsymbol{H}_{q}+$ $\frac{\left(\omega_{0}^{q}\right)^{2}}{c^{2}} \boldsymbol{H}_{q}=0$ for the localized volume and surface fields into

$\boldsymbol{H}_{q^{\prime}}^{*} \cdot \sum_{q} \nabla_{Z}^{2} C_{q}(z) \boldsymbol{H}_{q}+\boldsymbol{H}_{q^{\prime}}^{*} \cdot \sum_{q} \nabla_{\perp}^{2} C_{q}(z) \boldsymbol{H}_{q}+\left(\frac{\omega}{c}\right)^{2} \boldsymbol{H}_{q^{\prime}}^{*} \cdot$

$\sum_{q} C_{q}(z) \boldsymbol{H}_{q}=i \omega \varepsilon \boldsymbol{j}_{m} \cdot \boldsymbol{H}_{q \prime}^{*}$

gives

$\boldsymbol{H}_{q \prime}^{*} \cdot \sum_{q} \nabla_{z}^{2} C_{q}(z) \boldsymbol{H}_{q}+\boldsymbol{H}_{q}^{*} \cdot \sum_{q} C_{q}(z)\left(\frac{\omega^{2}-\omega_{0}^{2}}{c^{2}}\right) \boldsymbol{H}_{q}=i \omega \varepsilon \boldsymbol{j}_{m} \cdot \boldsymbol{H}_{q^{\prime}}^{*}$

where $q^{\prime}$ denotes the near-cut off volume mode. Imposing the orthogonality condition $\int_{S_{\perp}} \boldsymbol{H}_{q} \cdot \boldsymbol{H}_{q}^{*}, d \sigma=0$ if $q \neq q^{\prime}$ and integrating over the waveguide's cross-section $S_{\perp}$, where $d \sigma$ is the differential element of surface area, gives the normalized wave equation. Small diffractive and Ohmic losses are considered by introducing detuning parameters. Here, we introduce detuning between the variable frequency $\omega$ and the system eigenfrequencies $\omega_{0}$ and $\omega_{0}^{v, s}$ (where $\omega_{0}$ is the cut-off frequency if no corrugation is introduced i.e. pure volume wave as an ideal solution of Helmholtz equation while $\omega_{0}^{v, s}$ are eigenfrequencies of the volume and surface fields respectively if corrugations are introduced) is $\frac{\left(\omega^{2}-\omega_{0}^{2}\right)}{c^{2}}$ while the detuning between volume and surface modes is $\Delta=$ $\omega_{0}^{v}-\omega_{0}^{s} \cong c k_{z}^{v}$. We note that as corrugations are shallow $\omega_{0} \cong \omega_{0}^{v}$ while satisfying the Bragg resonance condition $\bar{k}_{z}=$ $k_{z}^{S}-k_{z}^{v}$ for the near cut-off $\left(k_{z}^{v} \cong 0\right) T M_{0, T}$ mode, requires that $\omega_{B}=\omega_{0}^{s}$. We define the Bragg detuning $\hat{\delta}=\frac{2\left(\omega_{B}-\bar{\omega}_{0}\right)}{c^{2}}$ in terms of the mean angular frequency, $\bar{\omega}_{0}=\frac{\omega_{0}^{v}+\omega_{0}^{s}}{2}$ and incorporate $\hat{\delta}$ into the normalized wave equation. We note that $\hat{\delta}$ can be complex and its imaginary part describes the losses.

$\nabla_{z}^{2} C_{q}^{v, s}(z)+\omega \hat{\delta} C_{q}^{v, s}(z) \mp \frac{\bar{\omega}_{0} \Delta}{c^{2}} C_{q}^{v, s}(z)=N_{v, s} \oint \boldsymbol{j}_{m} \cdot \boldsymbol{H}_{q}^{*} d \sigma$

$N_{v, s}=\frac{i \omega \varepsilon}{\oint_{S_{\perp}} \boldsymbol{H}_{q} \cdot \boldsymbol{H}_{q^{\prime}}^{*}, d \sigma}$ is the wave norm for the volume $(v)$ and surface $(s)$ modes, for which the "-" and "+" signs apply respectively. The integral $\oint_{S_{\perp}} \boldsymbol{H}_{q} \cdot \boldsymbol{H}_{q}^{*}, d \sigma$ denotes a closed surface integration with the boundaries defined by the smooth boundaries of the unperturbed waveguide.

Due to the structure's periodicity, it is possible to express the slowly varying surface field as a complex Fourier series of the form $C_{q}^{S}(z)=\sum_{n_{s}=-\infty}^{\infty} B_{n_{s}}(z) e^{i n_{s} \bar{k}_{z} z}$ where $B_{n_{s}}$ is the amplitude of the surface field's $n_{s}$ harmonic. Likewise, the volume field can be written as a complex Fourier expansion using the approximation $k_{z, v} \cong \bar{k}_{z}=2 \pi / d_{z}$ which equates the volume field's longitudinal wavenumber to that of the structure and corresponds to the case of coherent, coupled eigenmode formation i.e. minimal detuning. Evaluating the second order partial differential terms and substituting into Eq. (3) yields wave equations for the volume Eq. (4a) and surface Eq. (4b) modes. $\sum_{n_{v}}\left(e^{i n_{v} \bar{k}_{z} z} \frac{\partial^{2} A_{n_{v}}(z)}{\partial z^{2}}+2 i n_{v} \bar{k}_{z} e^{i n_{v} \bar{k}_{z} z} \frac{\partial A_{n_{v}}(z)}{\partial z}-\right.$ $\left.n_{v}{ }^{2} \bar{k}_{z}^{2} A_{n_{v}}(z) e^{i n_{v} \bar{k}_{z} z}\right)+\omega \hat{\delta}\left(\sum_{n_{v}} A_{n_{v}}(z) e^{i n_{v} \bar{k}_{z} z}\right)-$ $\frac{\bar{\omega}_{0} \Delta}{c^{2}}\left(\sum_{n_{v}} A_{n_{v}}(z) e^{i n_{v} \bar{k}_{z} z}\right)=N_{v} \oint \boldsymbol{j}_{m} \cdot \boldsymbol{H}_{q^{\prime}}^{*} d \sigma$

$\sum_{n_{s}}\left(e^{i n_{s} \bar{k}_{z} z} \frac{\partial^{2} B_{n_{S}}(z)}{\partial z^{2}}+2 i n_{s} \bar{k}_{z} e^{i n_{s} \bar{k}_{z} z} \frac{\partial B_{n_{S}}(z)}{\partial z}-\right.$

$\left.n_{s}^{2} \bar{k}_{z}^{2} B_{n_{s}}(z) e^{i n_{s} \bar{k}_{z} z}+\right)+\omega \hat{\delta}\left(\sum_{n_{s}} B_{n_{s}}(z) e^{i n_{s} \bar{k}_{z} z}\right)-$

$\frac{\bar{\omega}_{0} \Delta}{c^{2}}\left(\sum_{n_{s}} B_{n_{s}}(z) e^{i n_{s} \bar{k}_{z} z}\right)=N_{s} \oint \boldsymbol{j}_{m} \cdot \boldsymbol{H}_{q^{\prime}}^{*} d \sigma$

where $A_{n_{v}}$ is the amplitude of the $n_{v}$ harmonic of the volume field. To evaluate the right-hand side of Eq. $(4 a, b)$ we must define $\boldsymbol{j}_{m} \cdot \boldsymbol{H}_{q}^{*}$. A cylindrical PSL with sinusoidal corrugation $l=r_{0}+\Delta r \cos (\bar{m} \varphi) \cos \left(\bar{k}_{z} z\right)$ and $\bar{m}$ azimuthal variations is equivalent to a smooth cylindrical waveguide when the magnetic surface current boundary condition $\boldsymbol{j}_{m}=\boldsymbol{n} \times$ $(\boldsymbol{\nabla}(\boldsymbol{l} \boldsymbol{E} \cdot \boldsymbol{n}))+i \omega \boldsymbol{l n} \times[\boldsymbol{n} \times \boldsymbol{H}]$ is satisfied. For planar geometry, $\bar{m} \varphi$ is substituted with $\bar{k}_{y} y$, where $\bar{k}_{y}=2 \pi / d_{y}$. Enforcing this condition gives $\boldsymbol{j}_{m} \cdot \boldsymbol{H}_{q}^{*}=i \omega l(z, \varphi)\left(\boldsymbol{E}_{q, n} \cdot \boldsymbol{E}_{q, n}^{*}+\boldsymbol{H}_{q, \tau}\right.$. $\left.\boldsymbol{H}_{q, \tau}^{*}\right)$ where $\boldsymbol{E}_{q, n}$ and $\boldsymbol{H}_{q, \tau}$ are the normal electric and tangential magnetic field components with complex conjugates $\boldsymbol{E}_{q, n}^{*}$ and $\boldsymbol{H}_{q, \tau}^{*}$. In the case of a $T M_{0, T}$ volume mode (with no normal electric field component) $\boldsymbol{E}_{q, n}=0$. Separating $\boldsymbol{H}_{q, \tau}$ into its volume and surface field constituents $\boldsymbol{H}_{q, \tau}=\boldsymbol{H}_{q, \tau}^{v}(r) C_{q}^{v}(z)+\boldsymbol{H}_{q, \tau}^{s}(r, \varphi) C_{q}^{S}(z)$ and expanding to include the full set of eigenmodes gives

$$
\begin{gathered}
\boldsymbol{j}_{m} \cdot \boldsymbol{H}_{q}^{*}=i \omega\left[r_{0}+\frac{\Delta r}{4}\left(e^{i \bar{m} \varphi}+e^{-i \bar{m} \varphi}\right)\left(e^{i \bar{k}_{z} z}+e^{-i \bar{k}_{z} z}\right)\right] \boldsymbol{H}_{q, \tau}^{*, v} . \\
\left(\boldsymbol{H}_{q, \tau}^{v}(r) \sum_{2} C_{q}^{v}(z)+\boldsymbol{H}_{q, \tau}^{S}(r) \cos \left(m_{s} \varphi\right) \sum_{n_{s}} B_{n_{s}}(z) e^{i n_{s} \bar{k}_{z} z}\right)
\end{gathered}
$$

The $\boldsymbol{j}_{m} \cdot \boldsymbol{H}_{q}^{*}$ term is now integrated over the cylindrical cross section $d \sigma$, where $N_{v, s}^{\prime} \oint \boldsymbol{j}_{m} \cdot \boldsymbol{H}_{q, \tau}^{*(v, s)} d \sigma=N_{v, s}^{\prime} \int_{0}^{2 \pi} r\left(\boldsymbol{j}_{m}\right.$. $\left.\boldsymbol{H}_{q}^{*}\right)\left.\right|_{r=r_{0}} d \varphi$ and $N_{v, s}^{\prime}=i \omega N_{v, s}$. One of the necessary criteria for volume and surface mode coupling is $\oint \boldsymbol{j}_{m} \cdot \boldsymbol{H}_{q}^{*} d \sigma \neq 0$. This requirement is satisfied by averaging over the period of fast oscillations from 0 to $2 \pi$, neglecting exponential (oscillating) terms that would otherwise integrate to zero.

A coupled dispersion equation [1,3] describing the structure's coupled eigenfield is derived

$\left(\omega_{e}^{2}-\Lambda^{2}\right)\left[\Lambda^{4}-2 \Lambda^{2}\left(2+\Gamma^{2}+\omega_{e}^{2}\right)+\left(2-\Gamma^{2}+\omega_{e}^{2}\right)^{2}\right]=$
$2 \alpha^{4}\left(2-\Gamma^{2}+\omega_{e}^{2}-\Lambda^{2}\right)$

where the normalized coupling coefficient $\alpha$ is defined as

$$
\alpha=\frac{\pi r_{0} \Delta r}{2} \sqrt{N_{v}^{\prime} N_{s}^{\prime}\left[\underline{H}_{q, \tau}^{*, v}\left(r_{0}\right) \cdot \underline{H}_{q, \tau}^{S}\left(r_{0}\right)\right]\left[\underline{H}_{q, \tau}^{*, S}\left(r_{0}\right) \cdot \underline{H}_{q, \tau}^{v}\left(r_{0}\right)\right]}
$$

and where $\Lambda$ is the normalised wave vector, $\omega_{e}=$ $\sqrt{\delta^{2}+2 \delta+\widetilde{\Delta}^{2}}$ is a variable angular frequency and $\delta=$ $(\omega-\Omega) / \Omega, \quad \widetilde{\Delta}=\Phi / \Omega, \quad \Gamma=2 \bar{k}_{z} c /\left(\left(\omega_{0}^{v}\right)^{2}+\left(\omega_{0}^{S}\right)^{2}\right) \quad$ are 'renormalized' detuning parameters with constants $\Phi, \Omega=$ $\sqrt{\left(\left(\omega_{0}^{s}\right)^{2} \mp\left(\omega_{0}^{v}\right)^{2}\right) / 2}$. Solving Eqs. (6) and (7) involves performing an integral mode calculation around the boundary cross section. Analytical dispersion diagrams can otherwise be obtained by treating $\alpha$ and $\Gamma$ as variable parameters. The coupling coefficient $\alpha$ can then be estimated by comparing the analytical dispersion diagrams with those obtained from numerical modelling using CST Microwave Studio. 


\section{CONCLUSIONS}

A theoretical analysis has been developed of the formation of frequency-locked coupled eigenmodes in electromagnetic structures, facilitated by two dimensional complex electrodynamic surfaces. This analysis provides a formula for the calculation of the coupling coefficient between the relevant volume modes and the surface modes. The coupling coefficient can also be estimated by comparing the analytical dispersion diagrams with those obtained from numerical modelling using CST Microwave Studio.

\section{ACKNOWLEDGMENT}

The authors thank I.V. Konoplev for helpful discussions.

\section{REFERENCES}

[1] N. S. Ginzburg, N. Y. Peskov, A.S. Sergeev, et al., "The use of a hybrid resonator consisting of one-dimensional and two-dimensional Bragg reflectors for generation of spatially coherent radiation in a coaxial free-electron laser," Phys. Plasmas, vol. 9, (6), pp. 2798-2802, June 2002.
[2] I. V. Konoplev, A. J. MacLachlan, C. W. Robertson, et al., "Cylindrical periodic surface lattice as a metadielectric: Concept of a surface-field Cherenkov source of coherent radiation," Phys. Rev. A, vol. 84, (1), art. no. 013826, July 2011.

[3] I. V. Konoplev, A. J. MacLachlan, C. W. Robertson, A. W. Cross, and A. D. R. Phelps, Cylindrical, periodic surface lattice-Theory, dispersion analysis, and experiment, Appl. Phys. Lett., vol. 101, art. no. 121111 , Sept. 2012.

[4] A. J. MacLachlan, C. W. Robertson, A. W. Cross, et al., "Volume and surface mode coupling experiments in periodic surface structures for use in mm-THz high power radiation sources," AIP Advances, vol. 8, (10), art. no. 105115, Oct. 2018.

[5] A. J. MacLachlan, C. W. Robertson, I. V. Konoplev, et al., "Resonant excitation of volume and surface fields on complex electrodynamic surfaces", Phys. Rev. Appl., vol. 11, art. no. 034034, Mar. 2019.

[6] A. J. MacLachlan, C. W. Robertson, K. Ronald, A. W. Cross, and A. D. R. Phelps, "Mode coupling in periodic surface lattice and metamaterial structures for $\mathrm{mm}$-wave and $\mathrm{THz}$ applications," $\mathrm{SN}$ Appl. Sci., vol. 1, (6), art. no. 613, 2019. 